\title{
A Diagnostic Score (DS) Is a Powerful Tool in Diagnosis of Acute Appendicitis in Elderly Patients With Acute Abdominal Pain
}

\author{
MAARET ESKELINEN $^{1 *}$, JANNICA MEKLIN ${ }^{1 *}$, KARI SYRJÄNEN $^{2,3}$ and MATTI ESKELINEN ${ }^{1}$ \\ ${ }^{1}$ Department of Surgery, Kuopio University Hospital and School of Medicine, \\ University of Eastern Finland, Kuopio, Finland; \\ ${ }^{2}$ Molecular Oncology Research Center, Barretos Cancer Hospital, Barretos, Brazil; \\ ${ }^{3}$ SMW Consultants, Ltd., Kaarina, Finland
}

\begin{abstract}
Background/Aim: Although acute appendicitis $(A A)$ in elderly patients is different from AA in younger patients, the accuracy of diagnostic scores (DSs) in detecting $A A$ is rarely considered. Patients and Methods: A cohort of 470 AAP (acute abdominal pain) patients older than 50 years, including 224 women (53.7\%) and 193 men (46.3\%), were included in the study. The most significant diagnostic predictors were used to construct DS formulas for $A A$ diagnosis with (Tax+) and without body temperature (Tax). Meta-analytical techniques were used to calculate the summary Se and Sp estimates for each data sets (historytaking, findings, and DS formulas). Results: In SROC analysis, the AUC values for $i$ ) symptoms ii) signs and tests iii) $D S_{\text {Tax- }}$ and iv) $D S_{\text {Tax+ }}$ were as follows: i) $A U C=0.658$ (95\%CI=0.601-0.709); ii) $A U C=0.751 \quad$ (95\%CI $=0.701$ $0.800)$, iii) $A U C=0.977$ (95\%CI=0.942-1.000), and for iv) $A U C=0.980$ (95\%CI=0.956-1.000). Using roccomp analysis for these AUC values, the differences were significant as follows: between i) and ii) $p=0.0358$; between i) and iii) $p<0.0001$; between i) and iv) $p<0.0001$; between ii) and iii) $p<0.0001$; between ii) and iv) $p<0.0001$; and between iii) and $i v) p=0.682$. Conclusion: Similar to younger $A A$ patients, the DS formula was superior to both the clinical
\end{abstract}

This article is freely accessible online.

*These Authors contributed equally to this study.

Correspondence to: Matti Eskelinen, MD, Ph.D., School of Medicine, University of Eastern Finland, Kuopio, P.O. Box 100, FI70029 KYS, Finland. Tel: +358 17173311, Fax: +358 17172611, GSM: +358 400969444, e-mail: matti.eskelinen@kuh.fi

Key Words: Acute appendicitis, acute abdominal pain, elderly patients, diagnostic score, body temperature, ROC, HSROC, diagnostic accuracy. history-taking and findings, and therefore, the use of DS should be an important part of the diagnostic decision tree of $A A$ also in the elderly patients presenting with AAP.

Acute appendicitis (AA) in elderly patients is in many respects different from $\mathrm{AA}$ in younger patients with acute abdominal pain (AAP). AA among elderly patients might have lower diagnostic accuracy, longer delay from onset of symptoms to admission, and therefore, higher AA perforation rate leading to higher complication and mortality rate. Ceresoli et al. (1) analysed an AA cohort of 16,544 patients and showed, that after adolescence, the incidence of AA decreases along with increasing age (1). In their study on AAP patients, Kraemer et al. (2) reported that $15 \%$ of the patients older than 50 years of age had a final diagnosis of AA, as compared to nearly $30 \%$ of AA among younger patients (2). However, along with the aging of the Western populations, AA among the elderly is likely to be more common in the future (1).

The lack of commonly accepted guidelines and diagnostic scoring (DS) specified for elderly patients with AA (3) encouraged us to evaluate the accuracy of the clinical diagnosis of AA among elderly patients. We designed the present study to assess the relative accuracy of i) a clinical history-taking, ii) clinical findings, as well as iii) the DS without body temperature (Tax-) and iv) the DS with body temperature $(\mathrm{Tax}+)$ in detecting clinically confirmed AA among the elderly patients with AAP.

\section{Patients and Methods}

Included in the present study were 470 elderly patients older than 50 years, of whom 224 were women $(53.7 \%)$ and 193 were men $(46.3 \%)$. The diagnosis of AA was confirmed by considering all clinical history-taking details, clinical findings and results of the laboratory tests together and following the diagnostic criteria of AA as previously described (4-8) (Tables I and II). 
Table I. The clinical history of the elderly patients ( $\geq 50$ years) with acute appendicitis versus other cause of abdominal pain.

\begin{tabular}{|c|c|c|c|c|c|c|}
\hline Clinical history variable & Positive endpoint & Negative endpoint & $\mathrm{TP}$ & $\mathrm{FN}$ & FP & $\mathrm{TN}$ \\
\hline 1. Location of initial pain & Upper left or right quadrants of abdomen & Other quadrants of abdomen & 18 & 35 & 134 & 230 \\
\hline 2. Location of pain at diagnosis & Right lower quadrant of abdomen & Other quadrants of abdomen & 46 & 7 & 51 & 313 \\
\hline $\begin{array}{l}\text { 3. Duration of pain: Duration } \\
\text { of pain at diagnosis }\end{array}$ & $\leq 48$ hours & $>48 \mathrm{~h}$ & 39 & 14 & 228 & 136 \\
\hline 4. Intensity of abdominal pain & Subjectively weak/moderate pain & Intolerable pain & 46 & 7 & 289 & 75 \\
\hline $\begin{array}{l}\text { 5. Progression of pain from } \\
\text { onset to diagnosis }\end{array}$ & Subjectively same or worse pain & Weaker pain than at the onset & 37 & 16 & 247 & 117 \\
\hline 6. Type of pain & Intermitted pain & Colicky or steady pain & 7 & 46 & 37 & 327 \\
\hline 7. Aggravating factors & No aggravating factors & $\begin{array}{l}\text { Movement, coughing, } \\
\text { respiration, food or other }\end{array}$ & 48 & 5 & 235 & 129 \\
\hline 8. Relieving factors & No relieving factors or lying still & Vomiting, food or antacids & 46 & 1 & 283 & 49 \\
\hline 9. Previous similar pain & No & Yes & 45 & 7 & 212 & 150 \\
\hline 10. Vertigo & No & Yes & 53 & 0 & 344 & 18 \\
\hline 11. Nausea & No & Yes & 26 & 27 & 122 & 242 \\
\hline 12. Vomiting & No & Yes & 33 & 20 & 171 & 190 \\
\hline 13. Appetite & No appetite & Normal appetite & 43 & 10 & 282 & 82 \\
\hline 14. Previous indigestion & No & Yes & 44 & 9 & 231 & 132 \\
\hline 15. Jaundice & No & Yes & 53 & 0 & 344 & 20 \\
\hline 16. Bowels & Normal & $\begin{array}{l}\text { Constipation, diarrhea, blood, } \\
\text { mucus, white or normal stools }\end{array}$ & 45 & 8 & 242 & 122 \\
\hline 17. Micturition & Normal & Abnormal & 50 & 3 & 328 & 36 \\
\hline 18. Drugs for abdominal pain & No & Yes & 53 & 0 & 336 & 28 \\
\hline 19. Previous abdominal surgery & No & Yes & 43 & 10 & 182 & 182 \\
\hline 20. Previous abdominal diseases & No & Yes & 45 & 8 & 249 & 115 \\
\hline 21. Use of alcohol & No & Yes & 53 & 0 & 353 & 11 \\
\hline
\end{tabular}

TP: True positive; FN: false negative; FP: false positive; TN: true negative.

Identifying the DS models. In the computation of the diagnostic score (DS), a multivariate logistic (stepwise) regression analysis (SPSS Statistics 26.0.0.1; IBM, Armonk, NY, USA) was used to disclose the variables with an independent predictive value. All the variables presented in Tables I and II were included in the analysis as binary data, e.g. $\mathrm{AA}=1$ and other diagnosis of $\mathrm{AAP}=0$. Using the coefficients of the regression model, a DS was built and its predictive value for AA was studied. The coefficient of the multivariate analysis showed the relative risk $\left(R R=e^{n}, n=\beta\right)$ of a patient with a given history-taking detail, clinical findings or test to have an AA.

The formula without body temperature count (Tax-). The formula without Tax in hierarchical summary receiving operating characteristic (HSROC) analysis is; $\mathrm{DS}_{\mathrm{Tax}-}=2.40 \times$ location of pain at diagnosis (positive endpoint $=1$, negative endpoint $=0$ ) $+3.67 \times$ tenderness (positive endpoint $=1$, negative endpoint $=0$ ) $+2.93 \times$ rigidity (positive endpoint $=1$, negative endpoint $=0$ ) -6.54 (Table III).

The formula with body temperature count (Tax+). The formula with Tax is; $\mathrm{DS}_{\mathrm{Tax}+}=1.95 \times$ location of pain at diagnosis (positive endpoint $=1$, negative endpoint $=0)+1.25 \times$ previous abdominal surgery (positive endpoint $=1$, negative endpoint $=0$ ) $+4.07 \times$ tenderness (positive endpoint $=1$, negative endpoint $=0$ ) $+2.96 \times$ rigidity (positive endpoint $=1$, negative endpoint $=0$ ) $+1.31 \times$ body temperature (positive endpoint $=1$, negative endpoint $=0)-8.12$ (Table IV).
Statistical analysis. The other statistical analyses were performed using STATA/SE version 16.1 (StataCorp, College Station, TX, USA). Statistical tests presented were two-sided, and $p$-value $<0.05$ was considered statistically significant. Using $2 \times 2$ tables, we calculated sensitivity (Se) and specificity (Sp) with $95 \%$ confidence intervals $(95 \% \mathrm{CI})$ for each clinical history-taking detail, finding or test, and using meta-analytical technique (metaprop) separate forest plots for Se and $\mathrm{Sp}$ were created for each set of data, including each diagnostic variable (as equivalent to "study ID"). We calculated the summary estimates of Se and $\mathrm{Sp}$, positive (LR+) and negative likelihood ratio (LR-) and diagnostic odds ratio (DOR), using a random effect bivariate model and fitted the HSROC curves, including all diagnostic variables in the $\mathrm{DS}_{\mathrm{Tax}-}$ and $\mathrm{DS}_{\mathrm{Tax}+}$ models, using the AA endpoint.

\section{Results}

Diagnostic performance of the clinical history-taking. The overall Se of the clinical history-taking for detecting AA was $83 \%(95 \% \mathrm{CI}=72-92 \%)$. Se was higher than $83 \%$ for 11 diagnostic symptoms. The five best clinical history-taking variables (relieving factors, vertigo, jaundice, drugs for abdominal pain and use of alcohol) showed $98-100 \%$ Se in diagnosis of AA (Figure 1). The overall Sp of the historytaking for detecting AA was 34\% (95\% CI=22-46\%) (Figure 2). Altogether, 10 symptoms showed Sp higher than $34 \%$. 
Eskelinen et al: Age-specific Diagnostic Score in Acute Appendicitis

Table II. The clinical signs and investigations of elderly patients ( $\geq 50$ years) with acute appendicitis versus other cause of abdominal pain.

\begin{tabular}{|c|c|c|c|c|c|c|}
\hline Clinical signs and investigations & Positive endpoint & Negative endpoint & $\mathrm{TP}$ & FN & FP & $\mathrm{TN}$ \\
\hline 1. Mood & Normal & Distressed or anxious & 47 & 6 & 294 & 70 \\
\hline 2. Colour & Normal or flushed & Jaundiced, pale or cyanosed & 52 & 1 & 328 & 36 \\
\hline 3. Abdominal movement & Poor/nil & Normal & 6 & 47 & 34 & 329 \\
\hline 4. Scar & No & Yes & 42 & 11 & 178 & 185 \\
\hline 5. Distension & No & Yes & 49 & 4 & 293 & 69 \\
\hline 6. Tenderness & Right lower quadrant of abdomen & Other quadrants of abdomen & 50 & 3 & 49 & 315 \\
\hline 7. Mass & No & Yes & 53 & 0 & 338 & 26 \\
\hline 8. Rebound & Yes & No & 48 & 5 & 146 & 218 \\
\hline 9. Guarding & Yes & No & 49 & 4 & 199 & 165 \\
\hline 10. Rigidity & Yes & No & 39 & 14 & 78 & 286 \\
\hline 11. Murphy's positive & No & Yes & 50 & 3 & 292 & 72 \\
\hline 12. Bowel sounds & Normal & Abnormal & 51 & 2 & 274 & 90 \\
\hline 13. Renal tenderness & No & Yes & 37 & 16 & 242 & 122 \\
\hline 14. Rectal digital tenderness & Abnormal & Normal & 26 & 27 & 45 & 318 \\
\hline 15. Body temperature & $\geq 37.1^{\circ} \mathrm{C}$ & $<37.1^{\circ} \mathrm{C}$ & 32 & 21 & 88 & 233 \\
\hline 16. Leucocyte count (LC) & $\geq 10,000 / \mathrm{mm}^{3}$ & $<10,000 / \mathrm{mm}^{3}$ & 31 & 16 & 104 & 165 \\
\hline 17. Urine & Normal & Haematuria or bacteriuria & 47 & 0 & 281 & 22 \\
\hline
\end{tabular}

TP: True positive; FN: false negative; FP: false positive; TN: true negative.

Table III. Diagnostic score for acute appendicitis (AA) in elderly patients ( $\geq 50$ years) without body temperature in the DS model shown at five different cut-off levels: DS I=-3.60, DS II=-1.21, DS III=-0.47, DS IV=DS values between -4.14 and -0.47 excluded, $n=128$ patients, DS V=DS values between -1.21 and -0.47 excluded, $n=43$ patients.

\begin{tabular}{|c|c|c|c|c|c|c|}
\hline Diagnostic score (DS) & Positive endpoint & Negative endpoint & $\mathrm{TP}$ & FN & FP & $\mathrm{TN}$ \\
\hline 1. Logistic model DST- I & AA & Other cause of AAP & 50 & 3 & 54 & 310 \\
\hline 2. Logistic model DST- II & AA & Other cause of AAP & 49 & 4 & 38 & 326 \\
\hline 3. Logistic model DST- III & AA & Other cause of AAP & 37 & 16 & 7 & 357 \\
\hline 4. Logistic model DST- IV & AA & Other cause of AAP & 37 & 1 & 4 & 244 \\
\hline 5. Logistic model DST- V & AA & Other cause of AAP & 37 & 4 & 7 & 326 \\
\hline
\end{tabular}

TP: True positive; FN: false negative; FP: false positive; TN: true negative; AAP: acute abdominal pain. Formula for DS: $2.40 \times$ location of pain at diagnosis (positive endpoint $=1$, negative endpoint $=0)+3.67 \times$ tenderness (positive endpoint $=1$, negative endpoint $=0)+2.93 \times$ rigidity $($ positive endpoint=1, negative endpoint $=0$ ) -6.54 .

Table IV. Diagnostic score for acute appendicitis (AA) in elderly patients ( $\geq 50$ years) with body temperature in the DS model shown at five different cut-off levels: DS VII =-1.97, DS VIII=-1.09, DS IX=-0.66, DS X=DS values between -1.97 and -0.66 excluded, $n=29$ patients, $D S X I=D S$ values between -1.97 and 0.46 excluded, $n=43$ patients.

\begin{tabular}{|c|c|c|c|c|c|c|}
\hline Diagnostic score (DS) & Positive endpoint & Negative endpoint & $\mathrm{TP}$ & FN & FP & $\mathrm{TN}$ \\
\hline 1. Logistic model DST+ VII & $\mathrm{AA}$ & Other cause of AAP & 50 & 3 & 29 & 292 \\
\hline 2. Logistic model DST+ VIII & AA & Other cause of AAP & 49 & 4 & 27 & 294 \\
\hline 3. Logistic model DST+ IX & AA & Other cause of AAP & 41 & 12 & 9 & 312 \\
\hline 4. Logistic model DST+ X & AA & Other cause of AAP & 41 & 3 & 9 & 292 \\
\hline 5. Logistic model DST+ XI & AA & Other cause of AAP & 34 & 3 & 2 & 292 \\
\hline
\end{tabular}

TP: True positive; FN: false negative; FP: False positive; TN: True negative; AAP: acute abdominal pain. Formula for DS: $1.95 \times$ location of pain at diagnosis (positive endpoint $=1$, negative endpoint $=0)+1.25 \times$ previous abdominal surgery $($ positive endpoint $=1$, negative endpoint $=0$ ) $+4.07 \times$ tenderness (positive endpoint $=1$, negative endpoint $=0)+2.96 \times$ rigidity (positive endpoint $=1$, negative endpoint $=0$ ) $+1.31 \times$ body temperature (positive endpoint $=1$, negative endpoint $=0$ ) -8.12 . 


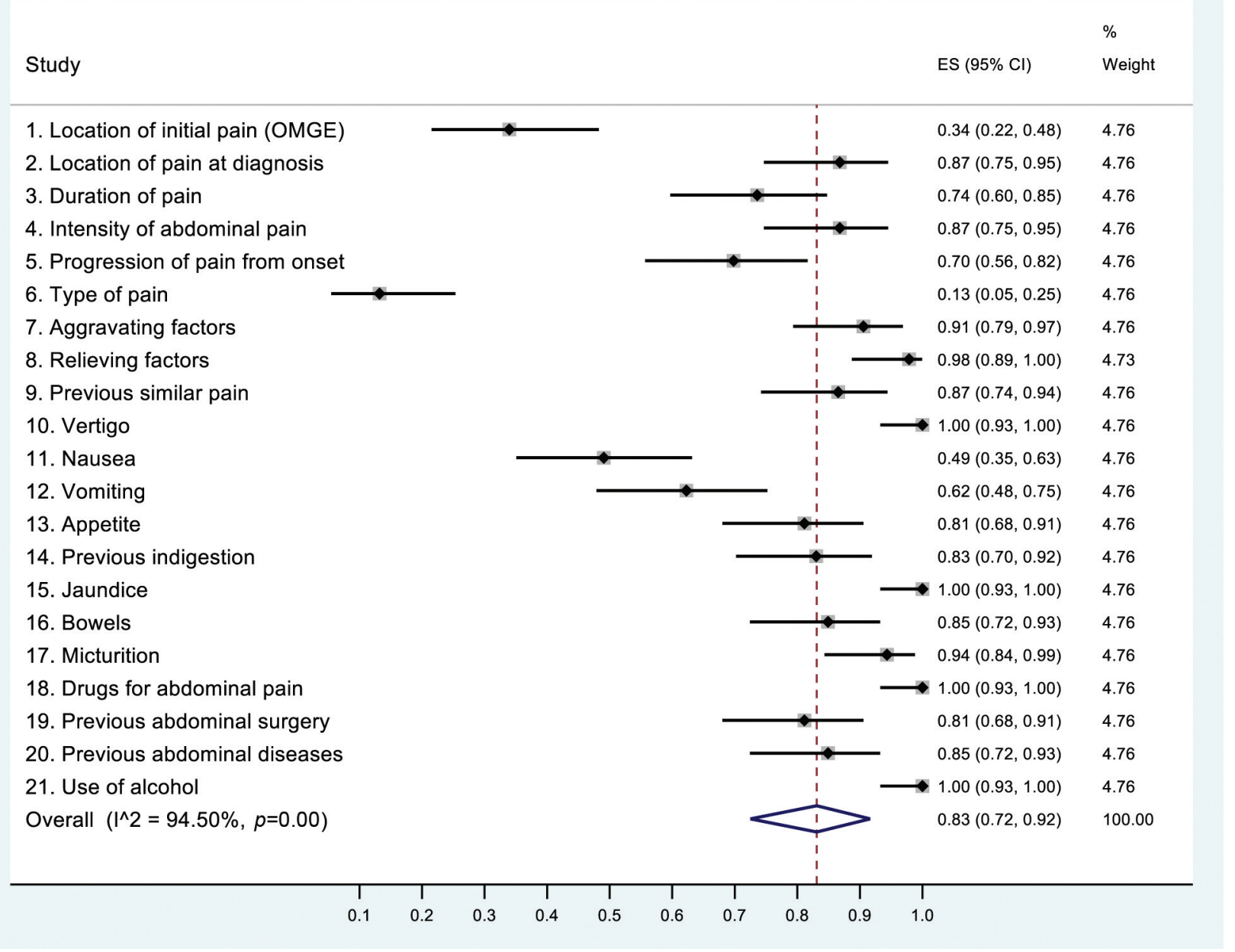

Figure 1. Sensitivities of the history-taking in acute appendicitis (random-effects model). ES: Estimated sensitivity; CI: confidence interval.

The five best symptoms of AA (location of initial pain, location of pain at diagnosis, type of pain, nausea, and vomiting) showed 53-90\% Sp (Figure 2).

Diagnostic performance of the clinical findings and tests. The overall Se of the diagnostic findings for detecting AA was $84 \%$ (95\% CI=72-93\%) (Figure 3), and 10 findings had Se exceeding $84 \%$. The five most accurate findings (colour, tenderness, mass, bowel sounds and urine) showed 94-100\% $\mathrm{Se}$ (Figure 3). The overall $\mathrm{Sp}$ of the findings was $45 \%$ $(95 \% \mathrm{CI}=29-61 \%)$ (Figure 4$)$, while 8 findings showed $\mathrm{Sp}$ higher than $45 \%$. The five most accurate findings (abdominal movement, tenderness, rigidity, rectal digital tenderness, and body temperature) showed $73-91 \%$ Sp (Figure 4).

Diagnostic performance of the DS without body temperature $\left(D S_{T a x_{-}}\right)$. The most important predictors of AA without Tax $(n=417)$ were location of pain at diagnosis, tenderness and rigidity, and used to construct the $\mathrm{DS}_{\mathrm{Tax}-}$ formula for $\mathrm{AA}$ diagnosis; $\mathrm{DS}_{\mathrm{Tax}=}=\mathrm{a}$ patient is admitted to the emergency room with abdominal pain; at diagnosis the pain was localized at the right lower quadrant of the abdomen (RLQ) $(1$ point $\times 2.40)$; clinical examination showed RLQ tenderness $(1$ point $\times 3.67)$, rigidity $(1$ point $\times 2.93)-$ constant value 6.54 . The best diagnostic performance level for $\mathrm{DS}_{\mathrm{Tax}-}$ formula (DS IV; $\mathrm{Se}=97 \%, \mathrm{~S} p=98 \%$ ) was reached when the patients with a $\mathrm{DS}_{\mathrm{Tax}-}$ value between -4.14 and 0.47 were considered as "undefined" patients for whom follow-up is required before the decision to operate $(n=128)$ (Figure 5). The DS model was tested at five different cut-off levels to disclose the best diagnostic performance in elderly patients (Figure 5). The $\mathrm{Se}$ and $\mathrm{Sp}$ of these five $\mathrm{DS}_{\mathrm{Tax}-}$ formulas were $90 \%(95 \% \mathrm{CI}=80-97 \%)$ and $95 \%(95 \% \mathrm{CI}=89-$ 99\%), respectively (Figures 5 and 6). Three of these 


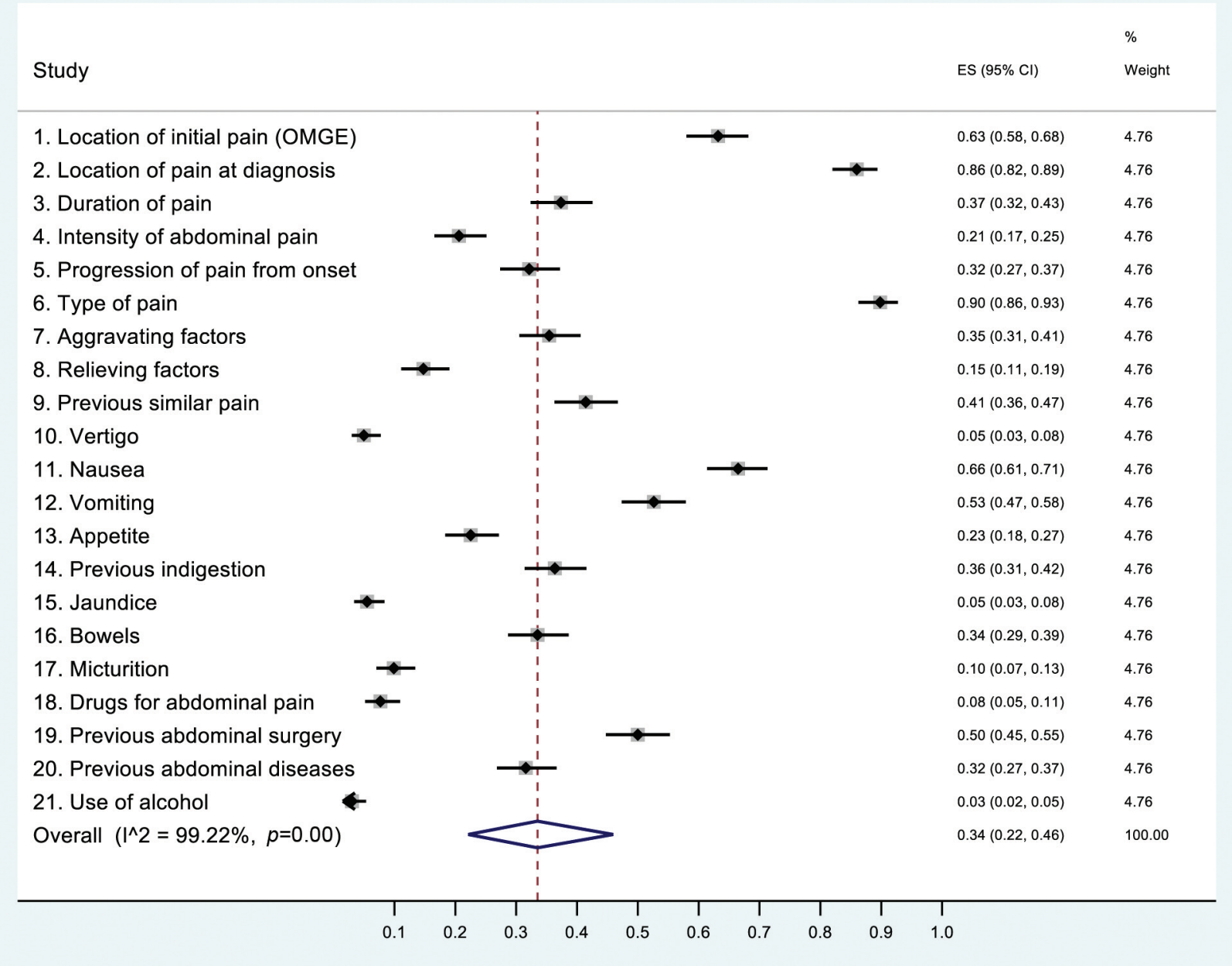

Figure 2. Specificities of the history-taking in acute appendicitis (random-effects model). ES: Estimated specificity; CI: confidence interval.

formulas showed $\mathrm{Se}>90 \%$ and three formulas had $\mathrm{Sp}>95 \%$. The best diagnostic $\mathrm{DS}_{\mathrm{Tax}-}$ formula in these elderly patients (formula DS IV, Figures 5 and 6) showed Se of $97 \%$ $(95 \% \mathrm{CI}=86-100 \%)$ and $\mathrm{Sp}$ of $98 \%(95 \% \mathrm{CI}=96-100 \%)$.

Diagnostic performance of the DS with body temperature $\left(D S_{\text {Tax+ }}\right)$. The significant independent predictors were used to build up the five different $\mathrm{DS}_{\mathrm{Tax}+}$ models. The Se and $\mathrm{Sp}$ of these five $\mathrm{DS}_{\mathrm{Tax}+}$ models were $90 \%$ (95\% CI=84-95\%) and $96 \%$ (95\% CI=92-98\%), respectively (Figures 7 and 8 ). Four formulas showed $\mathrm{Se}>90 \%$ and three formulas Sp over $96 \%$. The DS $\mathrm{Dax}_{\mathrm{Tax}}$ (formula DS IX, Figures 7 and 8) showed $\mathrm{Se}$ of $93 \%(95 \% \mathrm{CI}=81-99 \%)$ and $\mathrm{Sp}$ of $97 \%(95 \% \mathrm{CI}=94-$ 99\%) (Figures 7 and 8).

HSROC and comparison of the AUC values. STATA (metandiplot) was used to draw the HSROC curves to visualise the pooled overall diagnostic performance of the different DS formulas in detecting AA in elderly patients (Figures 9 and 10). In SROC analysis, the AUC values for i) clinical history-taking ii) diagnostic findings and tests iii) $\mathrm{DS}_{\mathrm{Tax}-}$ (Figure 11) and iv) $\mathrm{DS}_{\mathrm{Tax}+}$ (Figure 12) were as follows: i) $\mathrm{AUC}=0.658$ (95\% CI $=0.601-0.709)$; ii) $\mathrm{AUC}=0.751$ (95\% $\mathrm{CI}=0.701-0.800)$, iii) $\mathrm{AUC}=0.977(95 \% \mathrm{CI}=0.942-1.000)$, and for iv) $\mathrm{AUC}=0.980$ (95\% CI $=0.956-1.000)$. In roccomp analysis for the AUC values, the differences were significant as follows: between i) and ii) $p=0.0358$; between i) and iii) $p<0.0001$; between i) and iv) $p<0.0001$; between ii) and iii) $p<0.0001$; between ii) and iv) $p<0.0001$; and between iii) and iv) $p=0.682$.

\section{Discussion}

The clinical history-taking and findings in elderly versus younger AA patients. Our interest was to compare the 


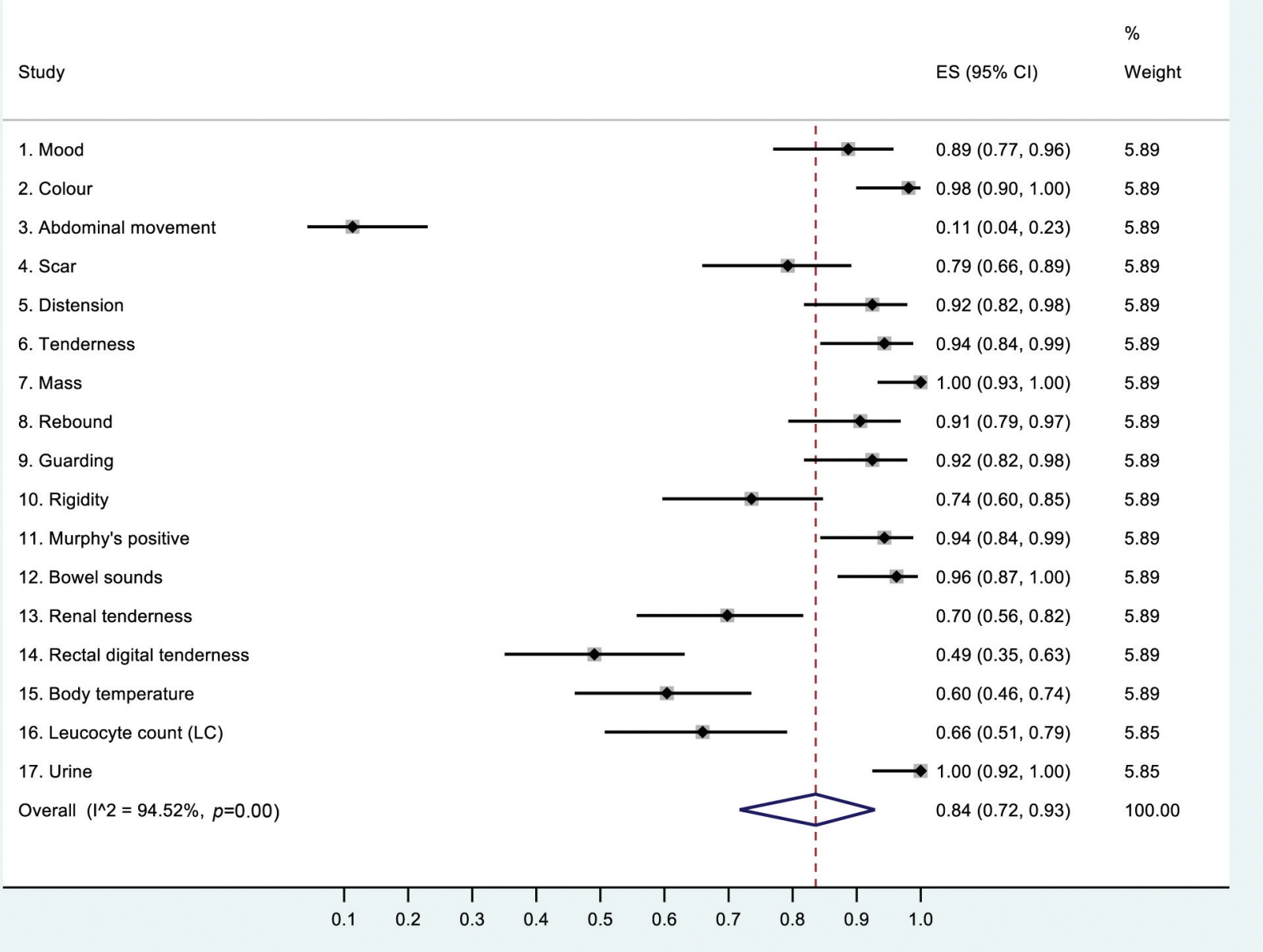

Figure 3. Sensitivities of the signs and tests in acute appendicitis (random-effects model). ES: Estimated sensitivity; CI: confidence interval.

performance of the clinical history-taking variables, findings and laboratory tests between the elderly AA patients and younger adult AA patients reported in our recent study (9), to examine whether the common clinical variables differ in elderly and younger adults. The Se of the history-taking in detecting AA in elderly $83 \%$ (95\% CI=72-92\%) was slightly higher than that detecting AA in younger female and male patients; $80 \%(95 \% \mathrm{CI}=67-90 \%)$ and $81 \%(95 \% \mathrm{CI}=66-92 \%)$, respectively. In addition, the overall $\mathrm{Sp}$ of the clinical history-taking in diagnosis of AA in elderly (34\%; $95 \% \mathrm{CI}=22-46 \%$ ) was slightly higher than that in younger adult female and male patients; $30 \%(95 \% \mathrm{CI}=19-42 \%)$ and $31 \%(95 \% \mathrm{CI}=20-43 \%)$, respectively. Instead, the overall Se of the findings and tests in detecting AA in the elderly was $84 \%(95 \% \mathrm{CI}=72-93 \%)$, which was slightly lower than that among the younger adult female and male patients; $86 \%$ $(95 \% \mathrm{CI}=79-92 \%)$ and $88 \%(95 \% \mathrm{CI}=82-94 \%)$, respectively.
However, the $\mathrm{Sp}$ of the clinical history-taking in diagnosis of AA in the elderly $(45 \% ; 95 \% \mathrm{CI}=29-61 \%)$ was significantly higher than that of the younger female and male patients; $34 \%(95 \% \mathrm{CI}=20-50 \%)$ and $34 \%(95 \% \mathrm{CI}=20-51 \%)$, respectively.

When the performance of the DS models was compared between elderly and younger adult AA patients, the trend was similar. The pooled Se of the DS models in diagnosis of AA in the elderly $(90 \%$; $95 \% \mathrm{CI}=80-97 \%)$ and in the younger female and male patients was very similar; $90 \%(95 \% \mathrm{CI}=85-$ $95 \%)$ and $93 \%(95 \% \mathrm{CI}=88-96 \%)$, respectively. Although, Se and $\mathrm{Sp}$ usually behave reciprocally, it was a surprise to find that the Sp of the DS in the elderly $(95 \% ; 95 \% \mathrm{CI}=89-99 \%)$ was significantly higher than that in the younger adult female and male patients; $85 \%(95 \% \mathrm{CI}=74-94 \%)$ and $84 \%$ (95\%CI $=74-92 \%)$, respectively. In spite of that, the AUC values based on HSROC analysis of the $\mathrm{DS}_{\mathrm{Tax}-}$ and $\mathrm{DS}_{\mathrm{Tax}+}$ 


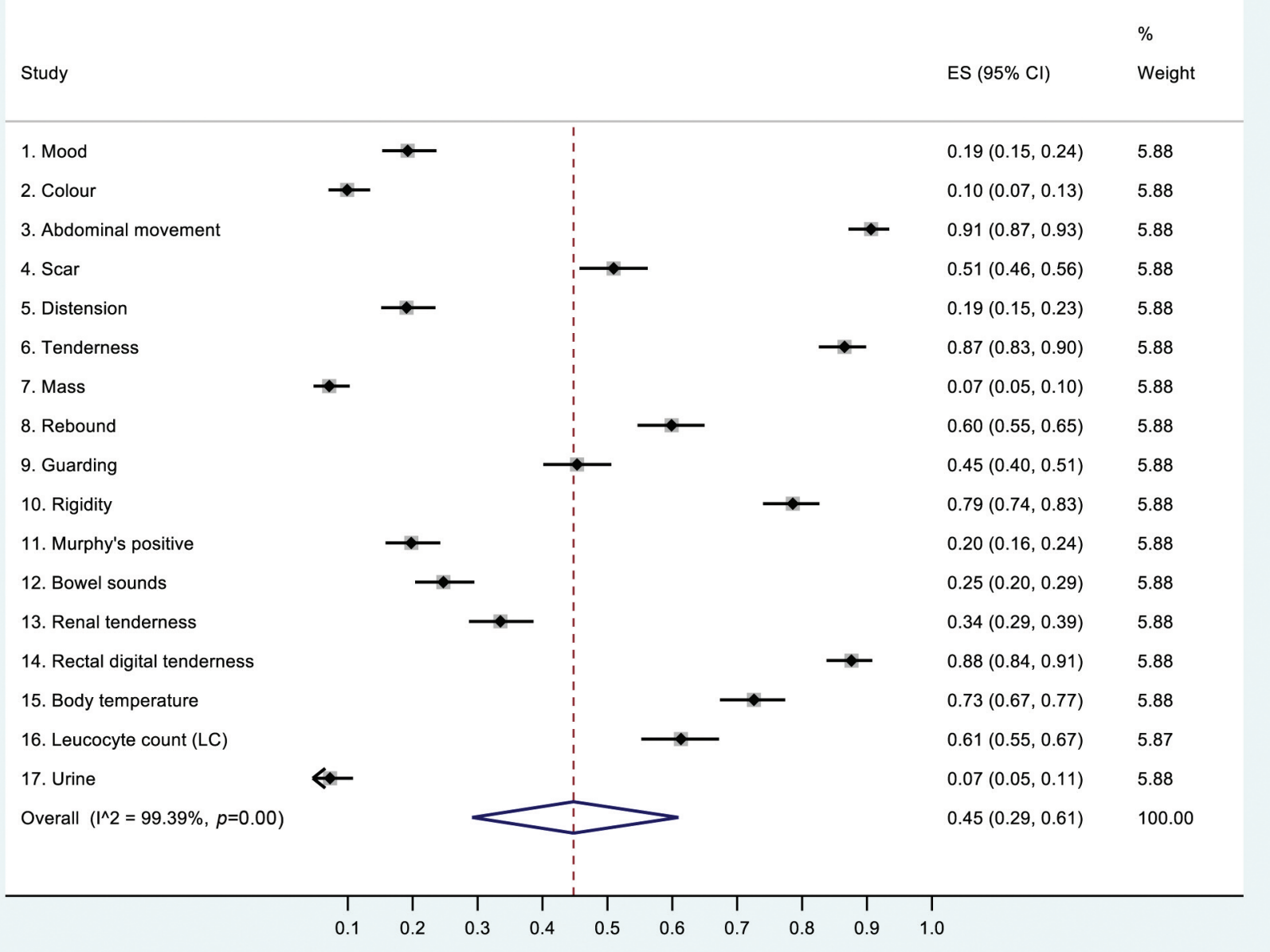

Figure 4. Specificities of the clinical signs and tests in acute appendicitis (random-effects model). ES: Estimated specificity; CI: confidence interval.

in the elderly [AUC $=0.977 \quad(95 \% \mathrm{CI}=0.942-1.000)$ and AUC $=0.980(95 \% \mathrm{CI}=0.956-1.000)$, respectively], and those in younger female $[0.953(95 \% \mathrm{CI}=0.923-0.969)]$ and male patients $[0.956(95 \% \mathrm{CI}=0.930-0.969)](9)$ were very similar, with no significant difference in the roccomp analysis.

Elderly patients $D S_{\text {Tax- }}$ and $D S_{\text {Tax+ }}$. AUC values based on HSROC comparison test showed that the diagnostic performance of the clinical findings was slightly better than that of the clinical history-taking details only $(p=0.0358)$. However, as measured by the AUC values, the DS model is superior to both i) the clinical history-taking and ii) findings and tests. Previous studies with a design similar to ours are scanty and only two retrospective studies have evaluated the applicability of the DS models in elderly AA population. Shchatsko et al. (9) used Alvarado score in 96 patients and suggested that elderly patients with Alvarado score between
5 and 10 had high risk of AA. Another retrospective study of 41 elderly patients (10) used Alvarado score with 0.969 AUC value. However, both these studies were retrospective $(9,10)$, and the lack of convincing evidence in elderly AA patients prompted the expert panel (3) not to recommend Alvarado score for diagnosis of AA in elderly patients.

The present study specifically focused on HSROC analysis and AUC value as the most important DS test characteristics. The main objective of the DS is to separate those elderly AA patients who do need urgent attention from those who don't need. Some previous studies $(2,9)$ have emphasized the value of Tax analysis in AA diagnosis, but Tax analysis has certain limitations, including i) variation in the definition of Tax cut-off, as well as ii) variations in the duration of abdominal pain in the time of Tax testing $(2,9)$. In earlier studies, the frequency of fever in the elderly patients with AA diagnosis has varied between $30-80 \%(2,9)$. There is 


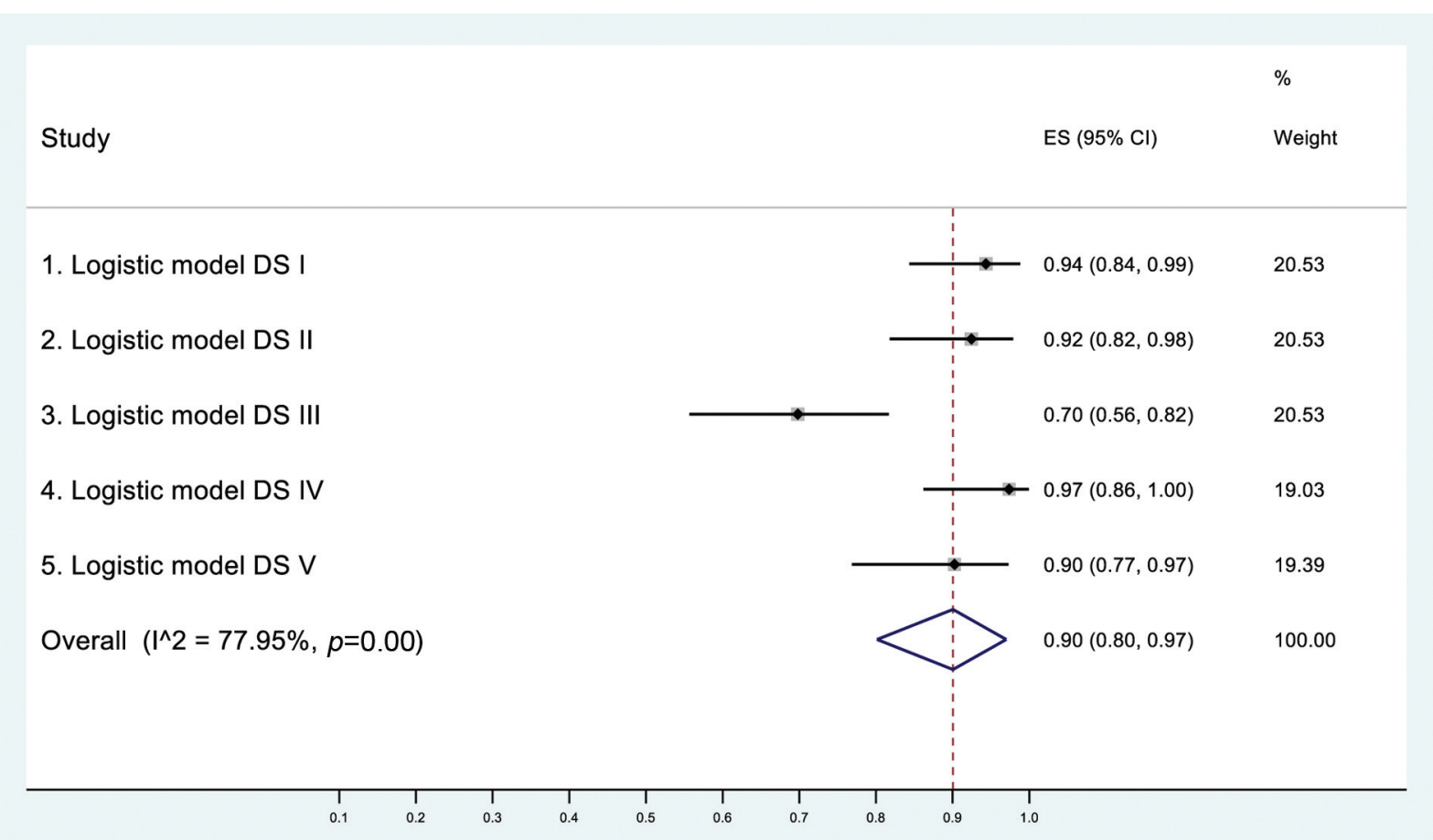

Figure 5. Sensitivities of diagnostic scores without body temperature $\left(D S_{\text {Tax- }}\right)$ at five different cut-off levels (DS I-V). ES: Estimated sensitivity; CI: confidence interval.

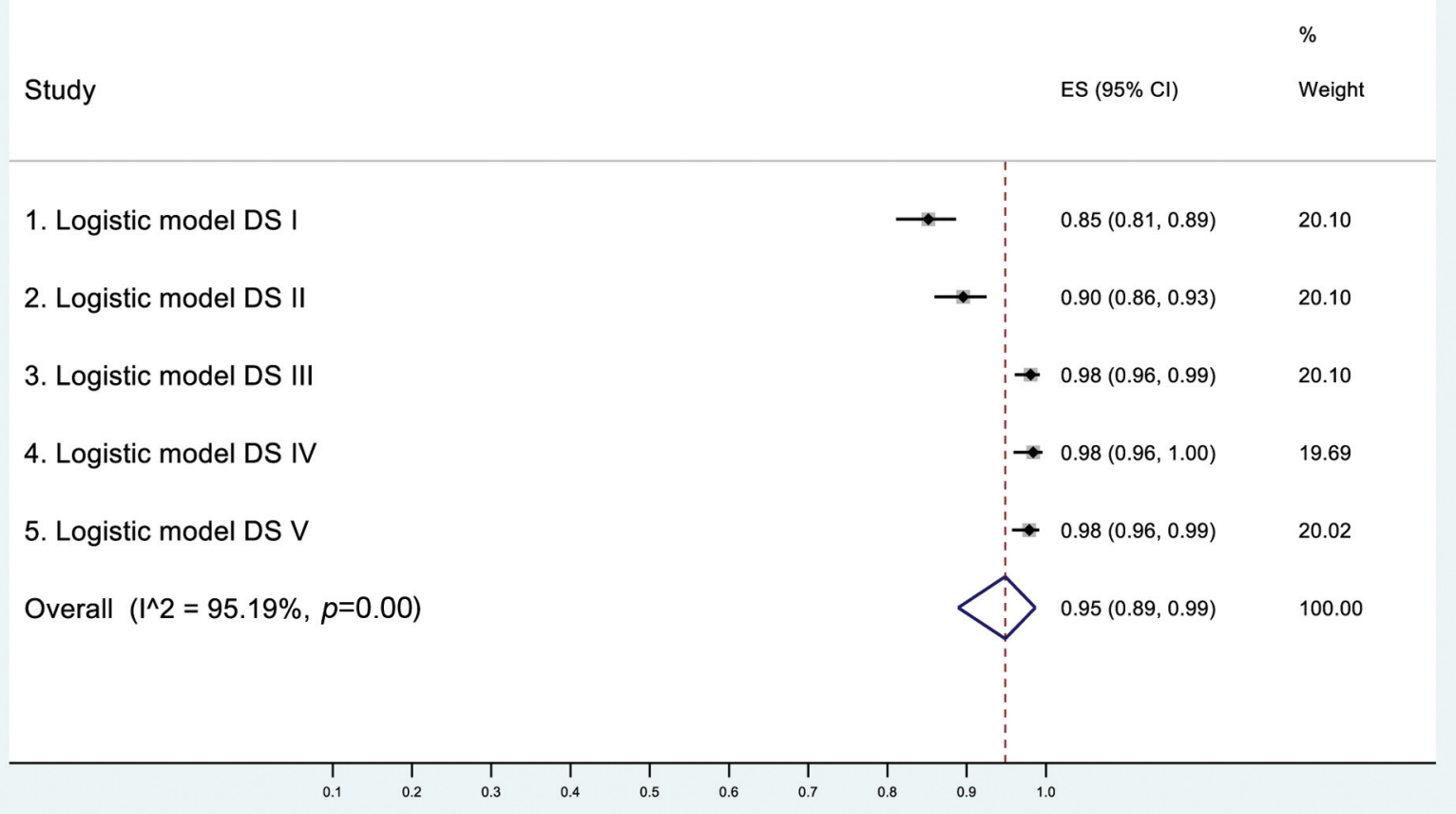

Figure 6. Specificities of diagnostic scores without body temperature $\left(D S_{\text {Tax- }}\right)$ at five different cut-off levels (DS I-V). ES: Estimated specificity; CI: confidence interval. 


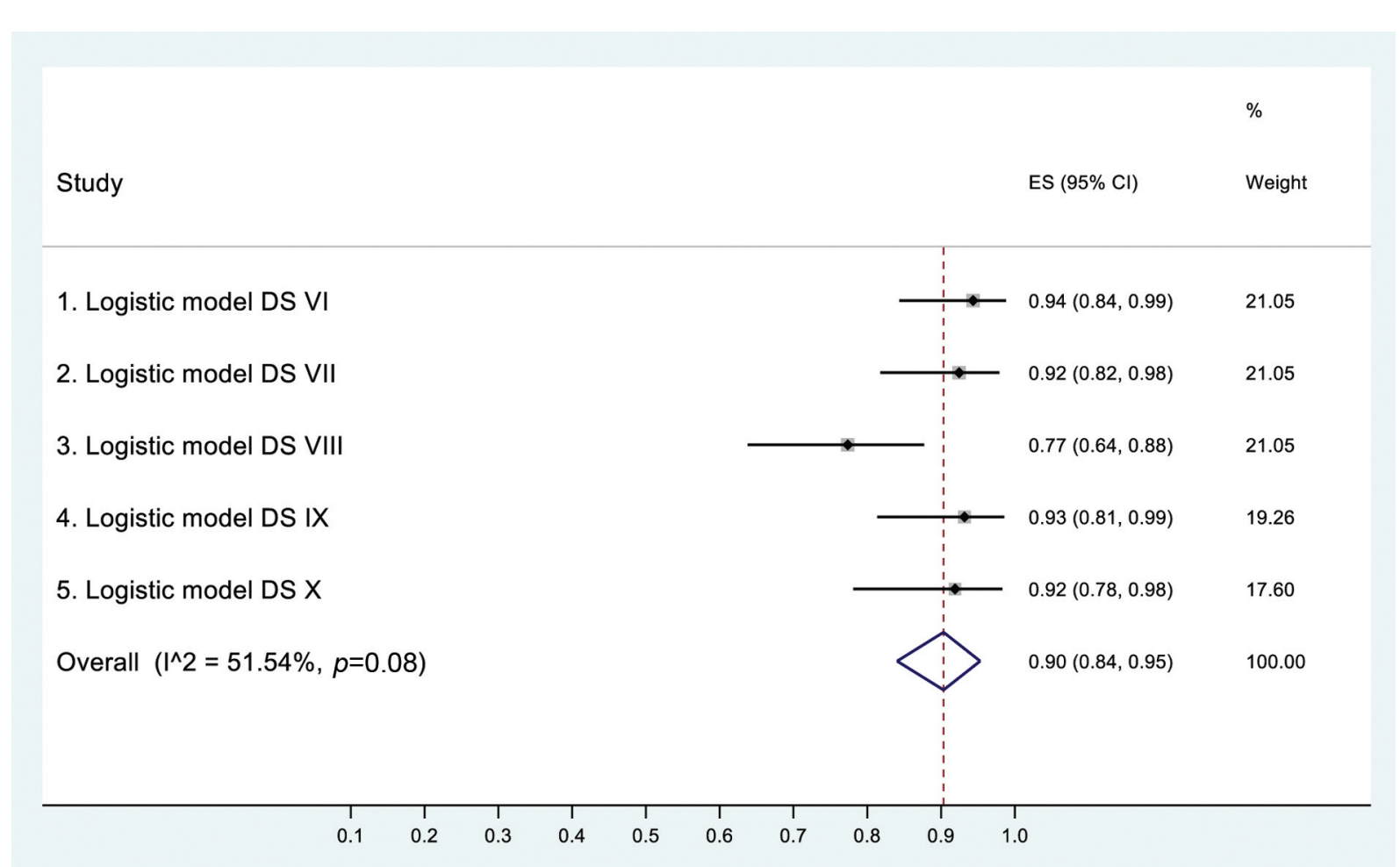

Figure 7. Sensitivities of diagnostic scores with body temperature $\left(D S_{\text {Tax+ }}\right)$ at five different cut-off levels $(D S$ VI-X). ES: Estimated sensitivity; CI: confidence interval.

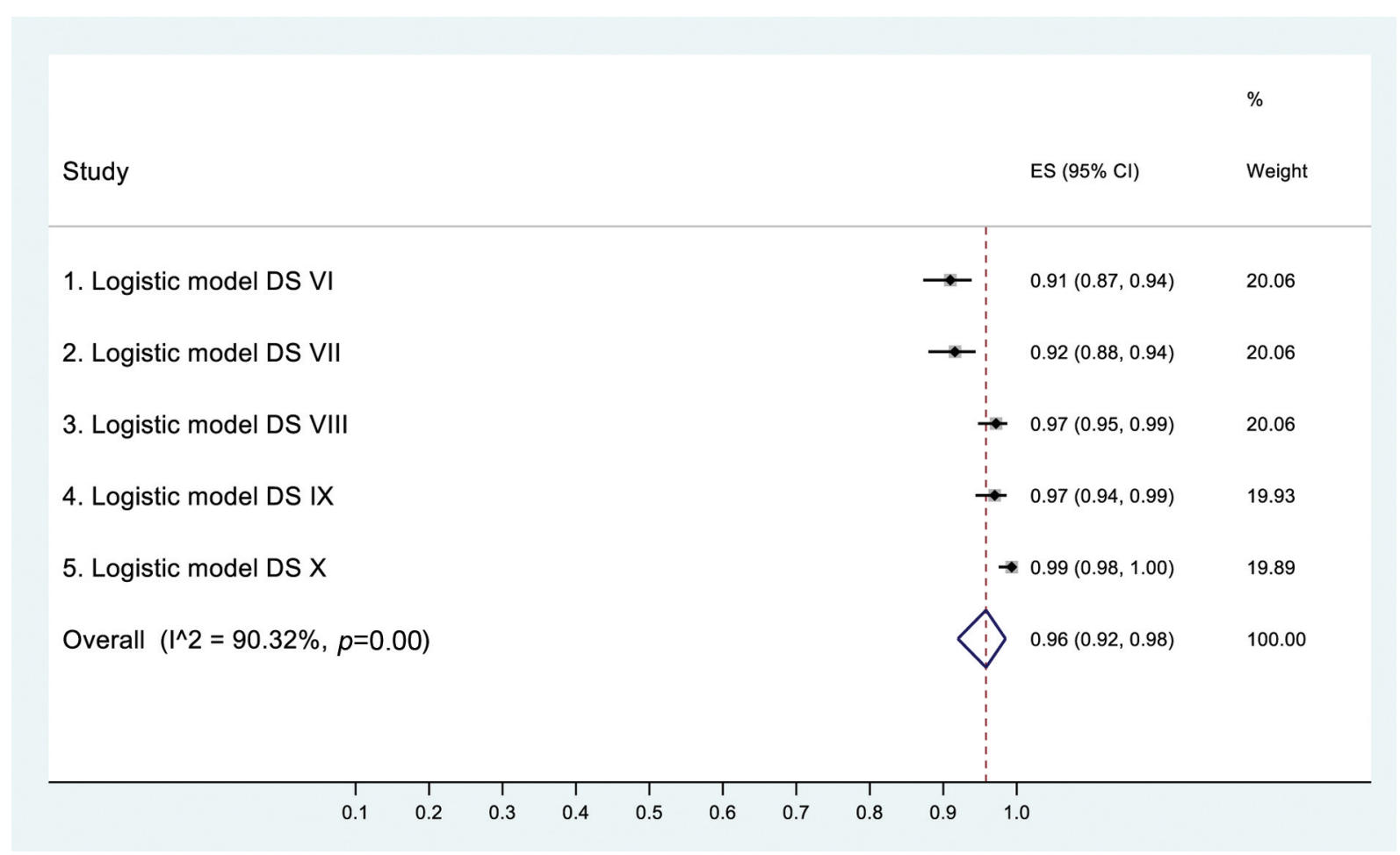

Figure 8. Specificities of diagnostic scores with body temperature $\left(D S_{T a x+}\right)$ at five different cut-off levels $(D S V I-X)$. ES: Estimated specificity; CI: confidence interval. 


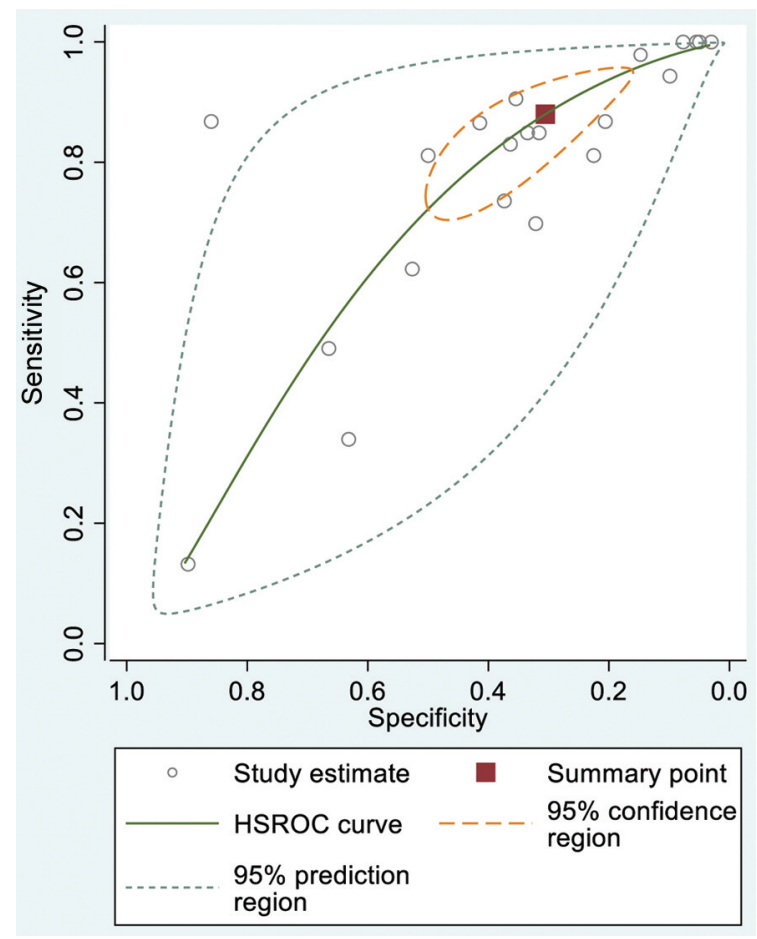

Figure 9. Hierarchical summary receiver operating characteristic (HSROC) curve of the history-taking in acute appendicitis.

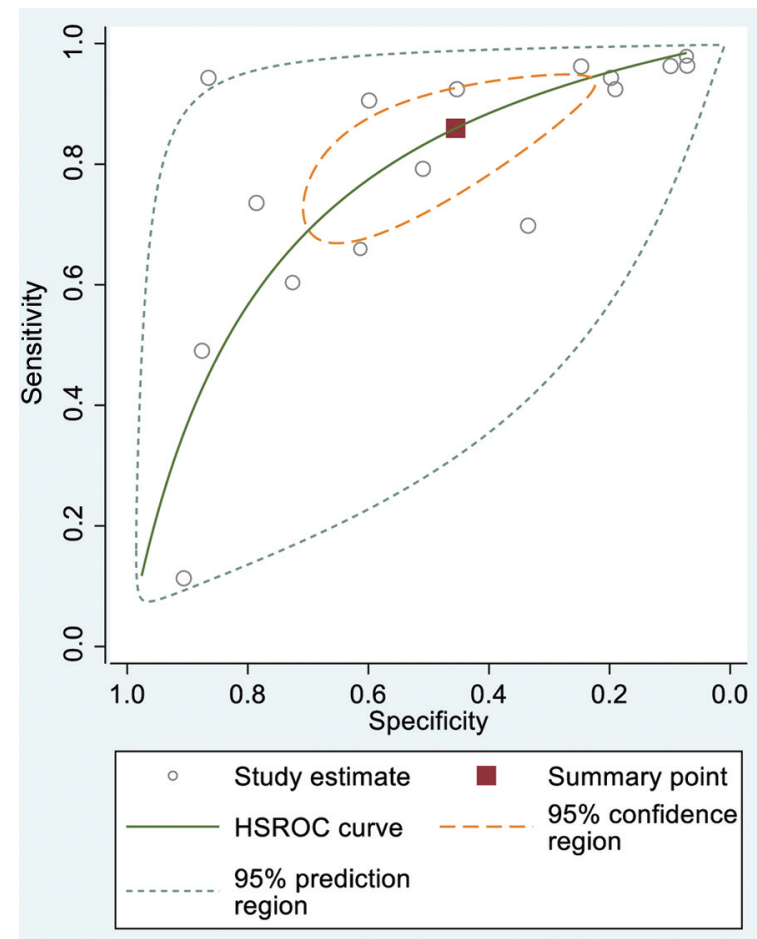

Figure 10. Hierarchical summary receiver operating characteristic (HSROC) curve of the clinical signs and tests in acute appendicitis.

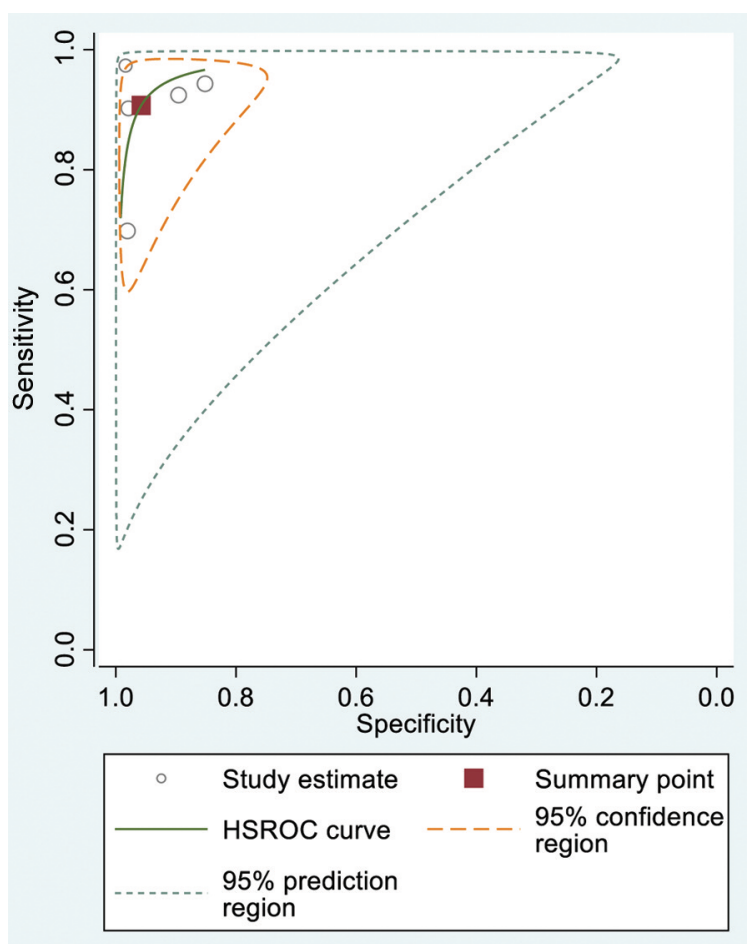

Figure 11. Hierarchical summary receiver operating characteristic (HSROC) curve of the five $D S_{\text {Tax }-}$.

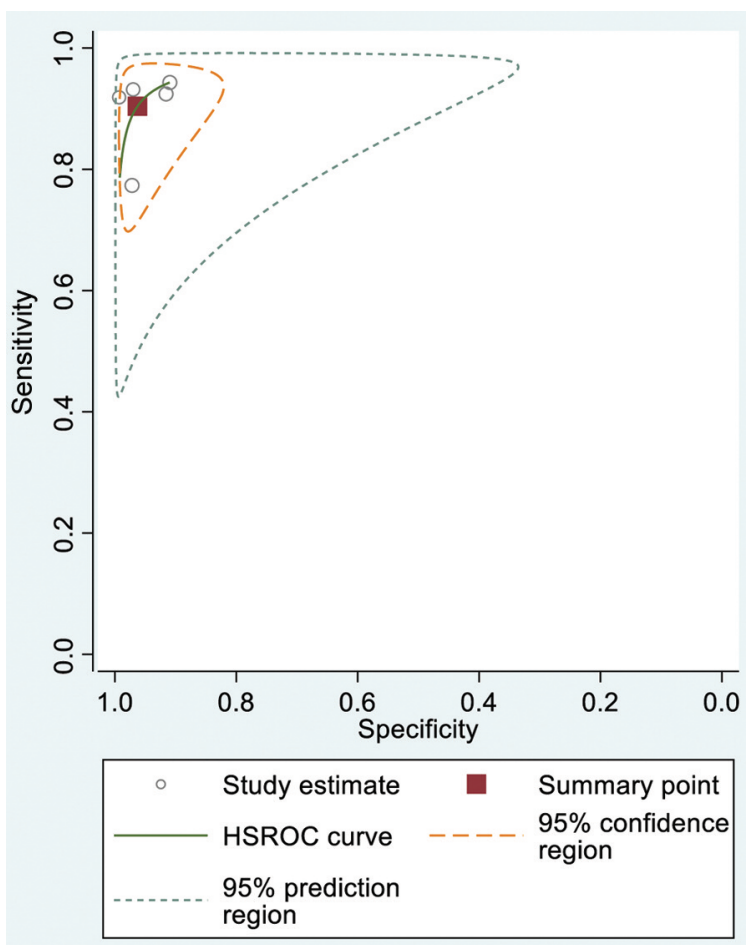

Figure 12. Hierarchical summary receiver operating characteristic (HSROC) curve of the five $\mathrm{DS}_{\mathrm{Tax}+}$. 
still controversy, whether the elderly AA patients with presentation of fever differ significantly from those without fever. The present study favours the lack of any such difference, because no statistically significant difference was established in the HSROC analysis between $\mathrm{DS}_{\mathrm{Tax}-}$ and $\mathrm{DS}_{\mathrm{Tax}+}$ formulas in diagnosis of AA in these elderly patients.

\section{Conclusion}

Although, some previous studies $(11,12)$ have reported that the diagnostic accuracy of AA is lower in the elderly patients as compared to younger AA patients, the results of our study do not support this statement. In contrast, the AUC value of DS for AA in the elderly patients is slightly higher than that in younger AA patients. Similar to the younger patients (6), the DS formula is superior to both the clinical history-taking and findings, and therefore the use of DS (with or without body temperature) should be an important part of the diagnostic decision tree of AA also in the elderly patients presenting with AAP.

\section{Conflicts of Interest}

The Authors report no conflicts of interest or financial ties in relation to this study.

\section{Authors' Contributions}

All Authors contributed to the collection and analysis of data, drafting, revising the manuscript, and read and approved the final manuscript.

\section{Acknowledgements}

The study was funded by the Päivikki and Sakari Sohlberg Foundation.

\section{References}

1 Ceresoli M, Zucchi A, Allievi N, Harbi A, Pisano M, Montori G, Heyer A, Nita GE, Ansaloni L and Coccolini F: Acute appendicitis: Epidemiology, treatment and outcomes- analysis of 16544 consecutive cases. World J Gastrointest Surg 8(10): 693699, 2016. PMID: 27830041. DOI: 10.4240/wjgs.v8.i10.693

2 Kraemer M, Franke C, Ohmann C, Yang Q and Acute Abdominal Pain Study Group. Acute appendicitis in late adulthood: incidence, presentation, and outcome. Results of a prospective multicenter acute abdominal pain study and a review of the literature. Langenbeck's Archives of Surgery 385(7): 470481, 2019. DOI: $10.1007 / \mathrm{s} 004230000165$
3 Fugazzola P, Ceresoli M, Agnoletti V, Agresta F, Amato B, Carcoforo P, Catena F, Chiara O, Chiarugi M, Cobianchi L, Coccolini F, De Troia A, Di Saverio S, Fabbri A, Feo C, Gabrielli F, Gurrado A, Guttadauro A, Leone L, Marrelli D, Petruzzelli L, Portolani N, Prete FP, Puzziello A, Sartelli M, Soliani G, Testini M, Tolone S, Tomasoni M, Tugnoli G, Viale P, Zese M, Ishay OB, Kluger Y, Kirkpatrick A and Ansaloni L: The SIFIPAC/WSES/SICG/SIMEU guidelines for diagnosis and treatment of acute appendicitis in the elderly (2019 edition). World J Emerg Surg 15(1): 19, 2020. PMID: 32156296. DOI: 10.1186/s13017-020-00298-0

4 Eskelinen M and Lipponen P: Usefulness of history-taking in non-specific abdominal pain: a prospective study of 1333 patients with acute abdominal pain in Finland. In Vivo 26(2): 335-339, 2012. PMID: 22351680

5 Meklin J, Eskelinen M, Syrjänen K and Eskelinen M: Leucocyte count does not improve the diagnostic performance of a diagnostic score (ds) in distinguishing acute appendicitis (aa) from nonspecific abdominal pain (NSAP). In Vivo 34(6): 33273339, 2020. PMID: 33144440. DOI: 10.21873/invivo.12171

6 Meklin J, Eskelinen M, Syrjänen K and Eskelinen M: Genderspecific performance of a diagnostic score in acute appendicitis. In Vivo 34(6): 3687-3703, 2020. PMID: 33144486. DOI: 10.21873/invivo. 12217

7 Eskelinen $\mathrm{M}$, Meklin J, Syrjänen $\mathrm{K}$ and Eskelinen $\mathrm{M}$ : Performance of a diagnostic score in confirming acute cholecystitis among patients with acute abdominal pain. Anticancer Research 40(12): 6947-6956, 2020. PMID: 33288589 DOI:10.21873/anticanres.14719

8 Eskelinen M, Meklin J, Syrjänen K and Eskelinen M: Pediatric acute appendicitis score in children with acute abdominal pain (AAP). Anticancer Research 41(1): 297-306, 2021. PMID: 33419824. DOI: 10.21873/anticanres.14776

9 Shchatsko A, Brown R, Reid T, Adams S, Alger A and Charles A: The utility of the alvarado score in the diagnosis of acute appendicitis in the elderly. Am Surg 83(7): 793-798, 2017. PMID: 28738954

10 Konan A, Hayran M, Kılıç YA, Karakoç D and Kaynaroğlu V: Scoring systems in the diagnosis of acute appendicitis in the elderly. Ulus Travma Acil Cerrahi Derg 17(5): 396-400, 2011. PMID: 22090323

11 Franz MG, Norman J and Fabri PJ: Increased morbidity of appendicitis with advancing age. Am Surg 61(1): 40-44, 1995. PMID: 7832380

12 Körner H, Söndenaa K, Söreide JA, Andersen E, Nysted A, Lende TH and Kjellevold KH: Incidence of acute nonperforated and perforated appendicitis: age-specific and sex-specific analysis. World J Surg 21(3): 313-317, 1997. PMID: 9015177. DOI: $10.1007 / \mathrm{s} 002689900235$
Received January 6, 2021

Revised January 29, 2021 Accepted February 1, 2021 